\title{
Meshless Singular Boundary Method for Nonlinear Sine-Gordon Equation
}

\author{
Yun Ji \\ Institute of Applied Electronics, Chongqing College of Electronic Engineering, Chongqing 401331, China \\ Correspondence should be addressed to Yun Ji; jy20023801@163.com
}

Received 8 May 2018; Accepted 12 June 2018; Published 5 July 2018

Academic Editor: Mariano Torrisi

Copyright (c) 2018 Yun Ji. This is an open access article distributed under the Creative Commons Attribution License, which permits unrestricted use, distribution, and reproduction in any medium, provided the original work is properly cited.

\begin{abstract}
A meshless method based on the singular boundary method is developed for the numerical solution of the time-dependent nonlinear sine-Gordon equation with Neumann boundary condition. In this method, by using a time discrete scheme to approximate the time derivatives, the time-dependent nonlinear problem is transformed into a sequence of time-independent linear boundary value problems. Then, the singular boundary method is used to establish the system of discrete algebraic equations. The present method is meshless, integration-free, and easy to implement. Numerical examples involving line and ring solitons are given to show the performance and efficiency of the proposed method. The numerical results are found to be in good agreement with the analytical solutions and the numerical results that exist in literature.
\end{abstract}

\section{Introduction}

The sine-Gordon equation is nonlinear and has drawn considerable attention as it comes up in a broad class of modeling situations such as nonlinear optics and solid state physics $[1,2]$. The solutions of nonlinear equations are important in mathematical physics and engineering. Many analytical methods [3-5] have been proposed to derive analytical solutions. It should be stressed that analytical solutions can only be gained in special cases. For instance, the study of analytical solutions of the sine-Gordon equation mostly focused on the undamped case.

In the past thirty years, a variety of numerical methods [6-15], such as the finite element method (FEM), the finite difference method (FDM), and the boundary element method (BEM), have been used to numerical simulation of the sine-Gordon equation. The success of these methods relies on the ability to construct and retain meshes of sufficient quality in the process of computation. In the past twenty years, meshless methods [16-20] have been developed to alleviate the meshing-related drawbacks. Up to now, a lot of meshless methods have been developed. Recently, some meshless methods [21-28] have been used to obtain the numerical solutions of the sine-Gordon equation.
The method of fundamental solutions (MFS) [29] is an extensively used meshless method. The approximate solution in the MFS is expressed as a linear combination of fundamental solutions. Compared with the BEM, the MFS does not need to compute singular integrals. However, to avoid the singularity of the fundamental solution, source points in the MFS are required to locate on a fictitious boundary outside the computational domain. Besides, the final system of algebraic equations in the MFS is sometimes ill-conditioned or nearly singular.

The singular boundary method (SBM) [30] is a recently developed meshless method. As in the MFS, the approximate solution in the SBM is also expressed as a linear combination of fundamental solutions. However, to isolate the singularity of fundamental solutions, the source intensity factor is proposed in the SBM. Thus, compared with the MFS, source points in the SBM are located on the real boundary instead of the fictitious boundary. The SBM has been used successfully for solving many boundary value problems [30-33]. Numerical results indicate that the SBM has much smaller condition number than the MFS.

In previous works, the SBM has only been used for problems governed by linear partial differential equations. The aim of this paper is to extend the SBM to the nonlinear 
sine-Gordon equation. The time derivatives in the nonlinear sine-Gordon equation are approximated by a time discrete scheme. Then, the SBM is used to establish the discrete algebraic equations. The present numerical method is truly meshless, free of integration, and easy to implement. Numerical examples are provided to illustrate the performance and capability of the method. Convergence studies are conducted to demonstrate the accuracy and efficiency of the present method.

The following discussions start with a description of the sine-Gordon equation. Then, detailed computational formulas of the SBM are presented for the sine-Gordon equation in Section 3. Finally, some numerical examples and conclusions are shown in Sections 4 and 5, respectively.

\section{Problem Description}

Let $\Omega$ be a two-dimensional bounded domain with boundary $\Gamma$. This paper is devoted to the numerical solution of the nonlinear sine-Gordon equation

$$
\frac{\partial^{2} u}{\partial t^{2}}+\beta \frac{\partial u}{\partial t}=\Delta u-\psi(\mathbf{x}) \sin (u), \quad \mathbf{x} \in \Omega, t>0
$$

with initial conditions

$$
\begin{aligned}
u(\mathbf{x}, 0)=f(\mathbf{x}), & \mathbf{x} \in \Omega \\
\left.\frac{\partial u(\mathbf{x}, t)}{\partial t}\right|_{t=0}=g(\mathbf{x}), & \mathbf{x} \in \Omega
\end{aligned}
$$

and boundary condition

$$
\frac{\partial u(\mathbf{x}, t)}{\partial \mathbf{n}}=\bar{q}(\mathbf{x}, t), \quad \mathbf{x} \in \Gamma, t>0
$$

where $u(\mathbf{x}, t)$ is the unknown wave displacement at position $\mathbf{x}$ and time $t, \psi(\mathbf{x}), f(\mathbf{x}), g(\mathbf{x})$, and $\bar{q}(\mathbf{x}, t)$ are known functions, and $\mathbf{n}=\left(n_{1}, n_{2}\right)$ is the unit outward normal on $\Gamma$. The function $\psi(\mathbf{x})$ can be explained as the Josephson current density, the function $f(\mathbf{x})$ is the wave modes or kinks, and the function $g(\mathbf{x})$ is the wave velocity. In addition, $\beta>0$ denotes the dissipative term. Equation (1) is the damped equation for $\beta>0$, and it is the undamped one for $\beta=0$.

\section{Numerical Computational Formulation}

3.1. Time Differencing. For discretization of time variable, the time derivatives can be approximated by the time-stepping scheme as

$$
\begin{aligned}
\frac{\partial^{2} u(\mathbf{x}, t)}{\partial t^{2}} & =\frac{u^{(k+1)}(\mathbf{x})-2 u^{(k)}(\mathbf{x})+u^{(k-1)}(\mathbf{x})}{\tau^{2}} \\
\frac{\partial u(\mathbf{x}, t)}{\partial t} & =\frac{u^{(k+1)}(\mathbf{x})-u^{(k-1)}(\mathbf{x})}{2 \tau}
\end{aligned}
$$

where $\tau>0$ denotes the step size of $t$ and

$$
u^{(k)}(\mathbf{x})=u(\mathbf{x}, k \tau), \quad k=0,1,2, \ldots
$$

In addition, $\Delta u$ can be approximated by the Crank-Nicolson scheme as

$$
\Delta u(\mathbf{x}, t)=\frac{1}{3}\left[\Delta u^{(k+1)}(\mathbf{x})+\Delta u^{(k)}(\mathbf{x})+\Delta u^{(k-1)}(\mathbf{x})\right]
$$

Then, substituting (5)-(8) into (1) yields

$$
\begin{aligned}
& \frac{u^{(k+1)}-2 u^{(k)}+u^{(k-1)}}{\tau^{2}}+\beta \frac{u^{(k+1)}-u^{(k-1)}}{2 \tau} \\
& =\frac{1}{3}\left[\Delta u^{(k+1)}+\Delta u^{(k)}+\Delta u^{(k-1)}\right]-\psi(\mathbf{x}) \sin u^{(k)}(\mathbf{x})
\end{aligned}
$$

Clearly, initial values $u^{(0)}$ and $u^{(-1)}$ are required in the iterative computation of (9). Using the initial conditions in (2) and (3), we have

$$
\begin{aligned}
u^{(0)}(\mathbf{x}) & =f(\mathbf{x}), \quad \mathbf{x} \in \Omega \\
u^{(-1)}(\mathbf{x}) & =u^{(1)}(\mathbf{x})-2 \tau g(\mathbf{x}), \quad \mathbf{x} \in \Omega
\end{aligned}
$$

In (9), letting $k=0$ and using (10) and (11) lead to

$$
\Delta u^{(1)}(\mathbf{x})-\frac{3}{\tau^{2}} u^{(1)}(\mathbf{x})=b^{(0)}(\mathbf{x})
$$

where

$$
\begin{aligned}
b^{(0)}(\mathbf{x})= & \tau \Delta g(\mathbf{x})-\frac{1}{2} \Delta f(\mathbf{x})-\frac{3}{\tau^{2}} f(\mathbf{x}) \\
& -\left(\frac{3}{\tau}-\frac{3 \beta}{2}\right) g(\mathbf{x})+\frac{3}{2} \psi(\mathbf{x}) \sin f(\mathbf{x})
\end{aligned}
$$

is known from the given functions.

When $k \geq 1$, we can write (9) as

$$
\begin{array}{r}
\Delta u^{(k+1)}(\mathbf{x})-\left(\frac{3}{\tau^{2}}+\frac{3 \beta}{2 \tau}\right) u^{(k+1)}(\mathbf{x})=b^{(k)}(\mathbf{x}), \\
k=1,2, \ldots
\end{array}
$$

where

$$
\begin{aligned}
b^{(k)}(\mathbf{x})= & -\Delta u^{(k)}(\mathbf{x})-\Delta u^{(k-1)}(\mathbf{x})-\frac{6 u^{(k)}(\mathbf{x})}{\tau^{2}} \\
& +\left(\frac{3}{\tau^{2}}-\frac{3 \beta}{2 \tau}\right) u^{(k-1)}(\mathbf{x}) \\
& +3 \psi(\mathbf{x}) \sin u^{(k)}(\mathbf{x})
\end{aligned}
$$

is known at the $(k+1)$ th iteration.

Finally, using (12) and (14), the original time-dependent problem (1)-(4) is reduced to the following time-independent problem:

$$
\begin{aligned}
\Delta u^{(k+1)}(\mathbf{x})-\lambda u^{(k+1)}(\mathbf{x}) & =b^{(k)}(\mathbf{x}), \quad \mathbf{x} \in \Omega \\
\frac{\partial u^{(k+1)}(\mathbf{x})}{\partial \mathbf{n}} & =\bar{q}(\mathbf{x}, k \tau), \quad \mathbf{x} \in \Gamma
\end{aligned}
$$

where $k=0,1,2, \ldots$ and $\lambda=3 / \tau^{2}$ for $k=0$ and $\lambda=3 / \tau^{2}+$ $3 \beta / 2 \tau$ for $k=1,2, \ldots$ 
3.2. Approximation of the Particular Solution. In (16), the constant $\lambda$ is positive. Thus, (16)-(17) compose a sequence of inhomogeneous problems for the modified Helmholtz equation. The solution can be represented as the sum of a particular solution $u_{p}^{(k+1)}$ and a homogeneous solution $u_{h}^{(k+1)}$; i.e.,

$$
u^{(k+1)}(\mathbf{x})=u_{h}^{(k+1)}(\mathbf{x})+u_{p}^{(k+1)}(\mathbf{x})
$$

where $u_{p}^{(k+1)}$ satisfies (16) as

$$
\Delta u_{p}^{(k+1)}(\mathbf{x})-\lambda u_{p}^{(k+1)}(\mathbf{x})=b^{(k)}(\mathbf{x}), \quad \mathbf{x} \in \Omega
$$

but not necessarily satisfies the boundary condition given in (17). Then, the homogeneous solution $u_{h}^{(k+1)}$ satisfies the following homogeneous equation:

$$
\Delta u_{h}^{(k+1)}(\mathbf{x})-\lambda u_{h}^{(k+1)}(\mathbf{x})=0, \quad \mathbf{x} \in \Omega
$$

with modified boundary condition

$$
\frac{\partial u_{h}^{(k+1)}(\mathbf{x})}{\partial \mathbf{n}}=\bar{q}(\mathbf{x}, k \tau)-\frac{\partial u_{p}^{(k+1)}(\mathbf{x})}{\partial \mathbf{n}}, \quad \mathbf{x} \in \Gamma
$$

To obtain the particular solution $u_{p}^{(k+1)}$, let $\left\{\mathbf{x}_{i}\right\}_{i=1}^{N} \subset \Gamma$ be a set of $N$ boundary nodes and $\left\{\mathbf{x}_{i}\right\}_{i=N+1}^{N+L} \subset \Omega$ be a set of $L$ interior nodes. Then, we can approximate the right-hand side of (19) by a finite linear combination of radial basis functions (RBFs) $\left\{\phi_{j}\right\}[34]$ as

$$
b^{(k)}(\mathbf{x})=\sum_{j=1}^{N+L} a_{j} \phi_{j}(\mathbf{x})
$$

where $a_{j}$ is the undetermined coefficient.

Collocating (22) for all nodes provides

$$
b^{(k)}\left(\mathbf{x}_{i}\right)=\sum_{j=1}^{N+L} a_{j} \phi_{j}\left(\mathbf{x}_{i}\right), \quad i=1,2, \ldots, N+L ;
$$

namely,

$$
\Phi \mathbf{a}=\mathbf{b}^{(k)}
$$

where

$$
\begin{aligned}
\boldsymbol{\Phi} & =\left(\begin{array}{cccc}
\phi_{1}\left(\mathbf{x}_{1}\right) & \phi_{2}\left(\mathbf{x}_{1}\right) & \cdots & \phi_{N+L}\left(\mathbf{x}_{1}\right) \\
\phi_{1}\left(\mathbf{x}_{2}\right) & \phi_{2}\left(\mathbf{x}_{2}\right) & \cdots & \phi_{N+L}\left(\mathbf{x}_{2}\right) \\
\vdots & \vdots & \ddots & \vdots \\
\phi_{1}\left(\mathbf{x}_{N+L}\right) & \phi_{2}\left(\mathbf{x}_{N+L}\right) & \cdots & \phi_{N+L}\left(\mathbf{x}_{N+L}\right)
\end{array}\right) \\
\mathbf{a} & =\left(a_{1}, a_{2}, \ldots, a_{N+L}\right)^{\mathrm{T}} \\
\mathbf{b}^{(k)} & =\left(b^{(k)}\left(\mathbf{x}_{1}\right), b^{(k)}\left(\mathbf{x}_{2}\right), \ldots, b^{(k)}\left(\mathbf{x}_{N+L}\right)\right)^{\mathrm{T}}
\end{aligned}
$$

Solving (24), we obtain

$$
\mathbf{a}=\boldsymbol{\Phi}^{-1} \mathbf{b}^{(k)}
$$

Finally, from (19) and (22) we obtain

$$
u_{p}^{(k+1)}(\mathbf{x})=\sum_{j=1}^{N+L} a_{j} \varphi_{j}(\mathbf{x})=\varphi^{\mathrm{T}}(\mathbf{x}) \mathbf{a}=\varphi^{\mathrm{T}}(\mathbf{x}) \Phi^{-1} \mathbf{b}^{(k)}
$$

$$
\mathbf{x} \in \Omega
$$

where $\varphi_{j}$ is related to the $\mathrm{RBF} \phi_{j}$ according to

$$
\Delta \varphi_{j}(\mathbf{x})-\lambda \varphi_{j}(\mathbf{x})=\phi_{j}(\mathbf{x}), \quad j=1,2, \ldots, N+L
$$

and

$$
\varphi^{\mathrm{T}}(\mathbf{x})=\left(\varphi_{1}(\mathbf{x}), \varphi_{2}(\mathbf{x}), \ldots, \varphi_{N+L}(\mathbf{x})\right)
$$

Many RBFs [34], such as multiquadric, Gaussians, linear distance functions, thin plate splines (TPS), and RBFs with compact supports, have been proposed and can be used for obtaining the particular solution. In this research, the following TPS is chosen:

$$
\phi_{j}(\mathbf{x})=r_{j}^{2}(\mathbf{x}) \ln r_{j}(\mathbf{x})
$$

And then, using the annihilator method [35], we have

$$
\begin{aligned}
\varphi_{j}(\mathbf{x})= & -\frac{1}{\lambda} r_{j}^{2}(\mathbf{x}) \ln r_{j}(\mathbf{x}) \\
& -\frac{4}{\lambda^{2}}\left[K_{0}\left(\sqrt{\lambda} r_{j}(\mathbf{x})\right)+\ln r_{j}(\mathbf{x})+1\right]
\end{aligned}
$$

where $r_{j}(\mathbf{x})=\left|\mathbf{x}-\mathbf{x}_{j}\right|$ and $K_{0}(\cdot)$ is the modified Bessel function of the second kind of order zero.

3.3. SBM for the Homogeneous Problem. In the SBM, the set of source points coincides with the set of collocation points. Then, we can express the solution of the homogeneous modified Helmholtz equation (20) as

$$
u_{h}^{(k+1)}(\mathbf{x})=\sum_{j=1}^{N} c_{j}^{(k+1)} K_{0}\left(\sqrt{\lambda} r_{j}(\mathbf{x})\right)
$$

where the zeroth-order modified Bessel function $K_{0}(\cdot)$ is the fundamental solution of the modified Helmholtz operator $\Delta-\lambda,\left\{\mathbf{x}_{i}\right\}_{i=1}^{N} \subset \Gamma$ is the set of source points, and $c_{j}^{(k+1)}$ is the unknown coefficient.

From (34) we have

$$
\frac{\partial u_{h}^{(k+1)}(\mathbf{x})}{\partial \mathbf{n}_{\mathbf{x}}}=\sum_{j=1}^{N} c_{j}^{(k+1)} \frac{\partial}{\partial \mathbf{n}_{\mathbf{x}}} K_{0}\left(\sqrt{\lambda} r_{j}(\mathbf{x})\right)
$$

where

$$
\begin{aligned}
& \frac{\partial}{\partial \mathbf{n}_{\mathbf{x}}} K_{0}\left(\sqrt{\lambda} r_{j}(\mathbf{x})\right) \\
& \quad=-\sqrt{\lambda} \frac{\left(\mathbf{x}-\mathbf{x}_{j}\right) \cdot \mathbf{n}_{\mathbf{x}}}{r_{j}(\mathbf{x})} K_{1}\left(\sqrt{\lambda} r_{j}(\mathbf{x})\right)
\end{aligned}
$$


and $K_{1}(\cdot)$ denotes the modified Bessel function of the second kind of order one.

To obtain the unknown coefficient $c_{j}^{(k+1)}$, using (34) and (35) and collocating the boundary condition given in (21) at $\mathbf{x}_{i}(i=1,2, \ldots, N)$ yield

$$
\begin{aligned}
& \sum_{j=1}^{N} c_{j}^{(k+1)} \frac{\partial}{\partial \mathbf{n}_{\mathbf{x}_{i}}} K_{0}\left(\sqrt{\lambda} r_{j}\left(\mathbf{x}_{i}\right)\right) \\
& \quad=\bar{q}\left(\mathbf{x}_{i}, k \tau\right)-\frac{\partial u_{p}^{(k+1)}\left(\mathbf{x}_{i}\right)}{\partial \mathbf{n}_{\mathbf{x}_{i}}}, \quad \text { for all } \mathbf{x}_{i} \in \Gamma
\end{aligned}
$$

Equation (37) contains $N$ linear algebraic equations, from which we can gain $N$ unknowns $c_{j}^{(k+1)}$. However, when source points $\mathbf{x}_{j}$ and collocation points $\mathbf{x}_{i}$ coincide, the singularity of the modified Bessel function $K_{0}(\cdot)$ occurs, and then the value of this function and its normal derivative can not be obtained directly. To isolate the singularity, the source intensity factor is proposed. Then, the SBM interpolation formula for (20) can be expressed as

$$
\begin{aligned}
& \sum_{j=1, j \neq i}^{N} c_{j}^{(k+1)} \frac{\partial}{\partial \mathbf{n}_{\mathbf{x}_{i}}} K_{0}\left(\sqrt{\lambda} r_{j}\left(\mathbf{x}_{i}\right)\right)+c_{i}^{(k+1)} Q_{i i} \\
& =\bar{q}\left(\mathbf{x}_{i}, k \tau\right)-\frac{\partial u_{p}^{(k+1)}\left(\mathbf{x}_{i}\right)}{\partial \mathbf{n}_{\mathbf{x}_{i}}}, \text { for all } \mathbf{x}_{i} \in \Gamma
\end{aligned}
$$

where $Q_{i i}$ is the source intensity factor corresponding to the singular term $\partial K_{0}\left(\sqrt{\lambda} r_{i}\left(\mathbf{x}_{i}\right)\right) / \partial \mathbf{n}_{\mathbf{x}_{i}}$.

The computational formulae of the source intensity factor $Q_{i i}$ are derived as follows. On the one hand, applying the subtracting and adding-back technique [36], from (35) we have

$$
\begin{aligned}
& \frac{\partial u_{h}^{(k+1)}\left(\mathbf{x}_{i}\right)}{\partial \mathbf{n}_{\mathbf{x}_{i}}}=\sum_{j=1}^{N} c_{j}^{(k+1)} \frac{\partial}{\partial \mathbf{n}_{\mathbf{x}_{i}}} K_{0}\left(\sqrt{\lambda} r_{j}\left(\mathbf{x}_{i}\right)\right) \\
& =\sum_{j=1, j \neq i}^{N}\left(c_{j}^{(k+1)}-\frac{l_{j}}{l_{i}} c_{i}^{(k+1)}\right) \frac{\partial K_{0}\left(\sqrt{\lambda} r_{j}\left(\mathbf{x}_{i}\right)\right)}{\partial \mathbf{n}_{\mathbf{x}_{i}}} \\
& \quad+\frac{c_{i}^{(k+1)}}{l_{i}} \sum_{j=1}^{N} l_{j} \frac{\partial K_{0}\left(\sqrt{\lambda} r_{j}\left(\mathbf{x}_{i}\right)\right)}{\partial \mathbf{n}_{\mathbf{x}_{i}}}
\end{aligned}
$$

where $\mathbf{x}_{i}, \mathbf{x}_{j} \in \Gamma$ and $l_{i}$ is the half distance of the curve between $\mathbf{x}_{i-1}$ and $\mathbf{x}_{i+1}$. When $i=j$, the second term in the right-hand side of (39) has a singularity. After a lengthy deduction [31], we have

$$
\begin{aligned}
& \sum_{j=1}^{N} l_{j} \frac{\partial K_{0}\left(\sqrt{\lambda} r_{j}\left(\mathbf{x}_{i}\right)\right)}{\partial \mathbf{n}_{\mathbf{x}_{i}}} \\
& \quad=\sum_{j=1, j \neq i}^{N}\left(l_{j} \frac{\partial K_{0}\left(\sqrt{\lambda} r_{j}\left(\mathbf{x}_{i}\right)\right)}{\partial \mathbf{n}_{\mathbf{x}_{i}}}+l_{j} \frac{\partial \ln r_{j}\left(\mathbf{x}_{i}\right)}{\partial \mathbf{n}_{\mathbf{x}_{j}}}\right)
\end{aligned}
$$

Substituting this equation into (39) yields

$$
\begin{aligned}
\frac{\partial u_{h}^{(k+1)}\left(\mathbf{x}_{i}\right)}{\partial \mathbf{n}_{\mathbf{x}_{i}}}= & \sum_{j=1, j \neq i}^{N} c_{j}^{(k+1)} \frac{\partial K_{0}\left(\sqrt{\lambda} r_{j}\left(\mathbf{x}_{i}\right)\right)}{\partial \mathbf{n}_{\mathbf{x}_{i}}} \\
& +\frac{c_{i}^{(k+1)}}{l_{i}} \sum_{j=1, j \neq i}^{N} l_{j} \frac{\partial \ln r_{j}\left(\mathbf{x}_{i}\right)}{\partial \mathbf{n}_{\mathbf{x}_{j}}}
\end{aligned}
$$

Besides, collocating (35) at the boundary node $\mathbf{x}_{i}$ yields

$$
\begin{aligned}
\frac{\partial u_{h}^{(k+1)}\left(\mathbf{x}_{i}\right)}{\partial \mathbf{n}_{\mathbf{x}_{i}}}= & \sum_{j=1, j \neq i}^{N} c_{j}^{(k+1)} \frac{\partial}{\partial \mathbf{n}_{\mathbf{x}_{i}}} K_{0}\left(\sqrt{\lambda} r_{j}\left(\mathbf{x}_{i}\right)\right) \\
& +c_{i}^{(k+1)} Q_{i i}
\end{aligned}
$$

Therefore, from the above two equations we have

$$
Q_{i i}=\frac{1}{l_{i}} \sum_{j=1, j \neq i}^{N} l_{j} \frac{\partial \ln r_{j}\left(\mathbf{x}_{i}\right)}{\partial \mathbf{n}_{\mathbf{x}_{j}}}, \quad i=1,2, \ldots, N
$$

Finally, the unknown coefficient $c_{j}^{(k+1)}$ can be obtained from (38) by solving

$$
\begin{aligned}
& \sum_{j=1, j \neq i}^{N} c_{j}^{(k+1)} \frac{\partial}{\partial \mathbf{n}_{\mathbf{x}_{i}}} K_{0}\left(\sqrt{\lambda} r_{j}\left(\mathbf{x}_{i}\right)\right)+c_{i}^{(k+1)} Q_{i i} \\
& =\bar{q}\left(\mathbf{x}_{i}, k \tau\right)-\frac{\partial \varphi^{\mathrm{T}}\left(\mathbf{x}_{i}\right)}{\partial \mathbf{n}_{\mathbf{x}_{i}}} \Phi^{-1} \mathbf{b}^{(k)}, \quad \text { for all } \mathbf{x}_{i} \in \Gamma
\end{aligned}
$$

where we have used (29). Equation (44) can be expressed as

$$
\mathbf{A c}^{(k+1)}=\gamma^{(k)}+\mathbf{B b}^{(k)}
$$

where $c^{(k+1)}=\left(c_{1}^{(k+1)}, c_{2}^{(k+1)}, \ldots, c_{N}^{(k+1)}\right)^{\mathrm{T}}$ is an unknown vector, $\gamma^{(k)}$ is a known vector containing the given boundary values $\bar{q}\left(\mathbf{x}_{i}, k \tau\right), \mathbf{A}$ and $\mathbf{B}$ are $N \times N$ matrices, and $\mathbf{b}^{(k)}$ is a vector given in (27) and is known at the $(k+1)$ th iteration.

After solving (45) to obtain $c_{j}^{(k+1)}$, then, according to (18), (29), and (34), approximate solution of the original nonlinear problem (1)-(4) can be computed as

$$
\begin{aligned}
u^{(k+1)}(\mathbf{x}) & =u_{h}^{(k+1)}(\mathbf{x})+u_{p}^{(k+1)}(\mathbf{x}) \\
& =\sum_{j=1}^{N} c_{j}^{(k+1)} K_{0}\left(\sqrt{\lambda} r_{j}(\mathbf{x})\right)+\varphi^{\mathrm{T}}(\mathbf{x}) \Phi^{-1} \mathbf{b}^{(k)},
\end{aligned}
$$

$$
\mathbf{x} \in \Omega
$$

\section{Numerical Results}

4.1. Test Problem. The test problem is the following nonlinear sine-Gordon equation:

$$
\frac{\partial^{2} u}{\partial t^{2}}=\frac{\partial^{2} u}{\partial x_{1}^{2}}+\frac{\partial^{2} u}{\partial x_{2}^{2}}-\sin (u),
$$

$$
x_{1}, x_{2} \in(-7,7), t>0
$$




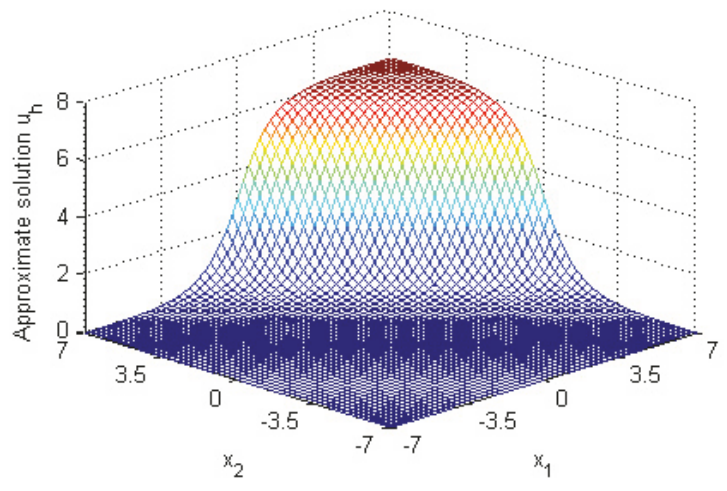

(a)

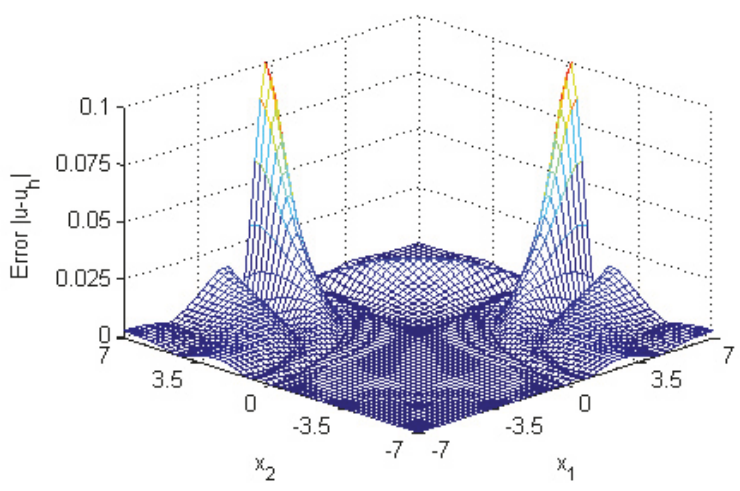

(b)

FIGURE 1: Graphs of (a) space-time and (b) error of the numerical solution up to $t=7$ with $\tau=0.01$ and $h=0.5$ for the test problem.

subject to initial conditions

$$
\begin{aligned}
& u\left(x_{1}, x_{2}, 0\right)=4 \tan ^{-1} \exp \left(x_{1}+x_{2}\right) \\
&\left.\frac{\partial u\left(x_{1}, x_{2}, t\right)}{\partial t}\right|_{t=0}=-\frac{4 \exp \left(x_{1}+x_{2}\right)}{1+\exp \left(2 x_{1}+2 x_{2}\right)} \\
& x_{1}, x_{2} \in(-7,7)
\end{aligned}
$$

and boundary conditions

$$
\begin{aligned}
& \frac{\partial u\left(x_{1}, x_{2}, t\right)}{\partial x_{1}}= \frac{4 \exp \left(x_{1}+x_{2}+t\right)}{\exp (2 t)+\exp \left(2 x_{1}+2 x_{2}\right)}, \\
& x_{1}= \pm 7, x_{2} \in(-7,7), t>0 \\
& \frac{\partial u\left(x_{1}, x_{2}, t\right)}{\partial x_{2}}=\frac{4 \exp \left(x_{1}+x_{2}+t\right)}{\exp (2 t)+\exp \left(2 x_{1}+2 x_{2}\right)}, \\
& x_{2}= \pm 7, x_{1} \in(-7,7), t>0
\end{aligned}
$$

The analytical solution is [21]

$$
u\left(x_{1}, x_{2}, t\right)=4 \tan ^{-1} \exp \left(x_{1}+x_{2}-t\right)
$$

Figure 1 gives the space-time graph of the numerical solution and the graph of the associated error. The results are obtained by using $\tau=0.01$ and $h=0.5$.

For the error estimation and convergence analysis, the following $L_{\infty}$ and root-mean-square (RMS) errors are used:

$$
\begin{aligned}
L_{\infty} & =\max _{1 \leq i \leq N_{s}}\left|u^{(i)}-u_{h}^{(i)}\right|, \\
\mathrm{RMS} & =\sqrt{\frac{1}{N_{s}} \sum_{i=1}^{N_{s}}\left(u^{(i)}-u_{h}^{(i)}\right)^{2}}
\end{aligned}
$$

where $N_{s}$ is the number of test points in $\Omega$ and $u^{(i)}$ and $u_{h}^{(i)}$ are the analytical and numerical values at the $i$-th test point, respectively.
$L_{\infty}$ and RMS errors at $t=1,3,5$, and 7 are plotted in Figure 2 for the SBM using $\tau=0.01$ and $h=0.25$. For comparison, the errors of the RBF method [21], the explicit method [6], and the moving Kriging-based meshless local Petrov-Galerkin (MK-MLPG) method [25] obtained by using $\tau=0.001$ and $h=0.25$ are also given. Comparing the errors of these methods confirms the good accuracy of the SBM.

Figure 3 gives the errors at times $t=1,3,5$, and 7 for the SBM against the nodal spacing $h$. In this analysis, $\tau=0.01$. Clearly, as the nodal spacing decreases, the error becomes smaller. In addition, the SBM possesses high experimentally convergence rate.

4.2. Line Soliton in an Inhomogeneous Medium. A line soliton for an inhomogeneity on large-area Josephson junction can be simulated by solving the sine-Gordon equation for $\beta=0$ and $[7-11,21]$

$$
\psi\left(x_{1}, x_{2}\right)=1+\operatorname{sech}^{2} \sqrt{x_{1}^{2}+x_{2}^{2}}
$$

and initial conditions

$$
\begin{aligned}
& f\left(x_{1}, x_{2}\right)=4 \tan ^{-1} \exp \left(\frac{x_{1}-3.5}{0.954}\right), \\
& g\left(x_{1}, x_{2}\right)=0.629 \operatorname{sech}\left(\frac{x_{1}-3.5}{0.954}\right)
\end{aligned}
$$

in the domain $-7 \leq x_{1}, x_{2} \leq 7$. The boundary condition is

$$
\frac{\partial u(\mathbf{x}, t)}{\partial \mathbf{n}}=0, \quad \mathbf{x} \in \Gamma, t \geq 0
$$

Figure 4 presents the results of this problem for $\tau=0.01$ and $h=0.5$ at $t=0,6,12$, and 18. In this figure, both the contours and surface plots of the ring soliton are given in terms of $\sin (u / 2)$. We can see that the soliton is moving in $x_{1}$-direction as a straight line soliton during the transmission through inhomogeneity. When $t=6$, we can observe a deformation in its straightness. Then, this movement seems to be impeded as $t$ tends to 12 . Finally, the soliton recovers its 


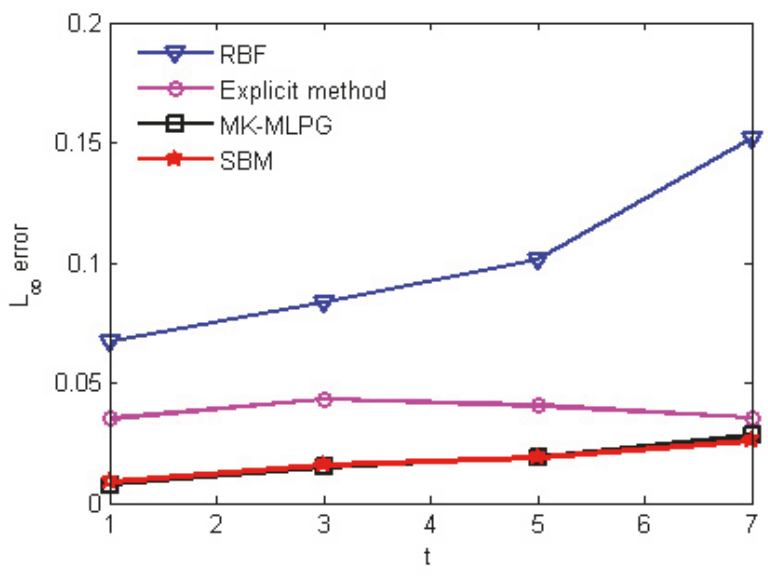

(a)

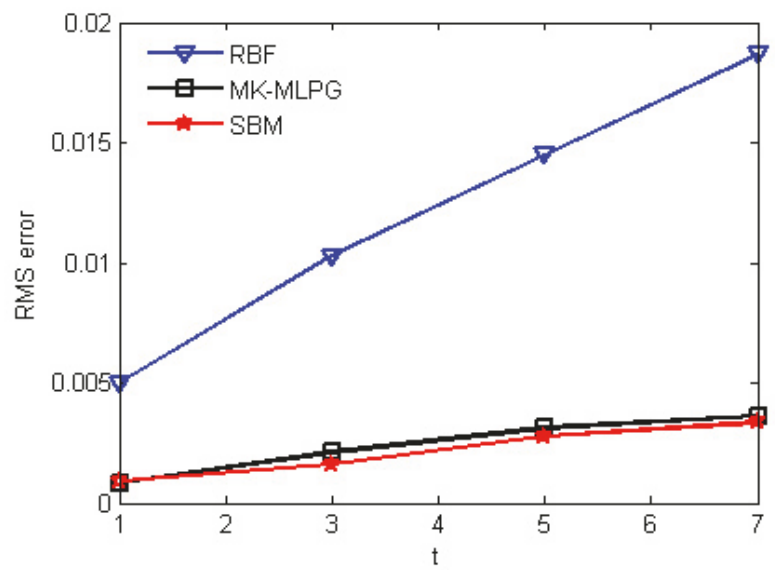

(b)

FIGURE 2: (a) $L_{\infty}$ error and (b) RMS error of the SBM and the RBF method for the test problem.

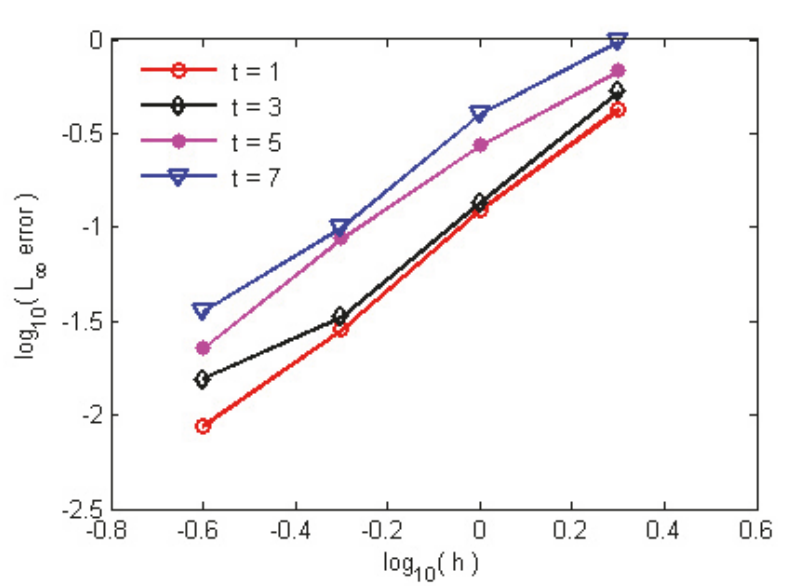

(a)

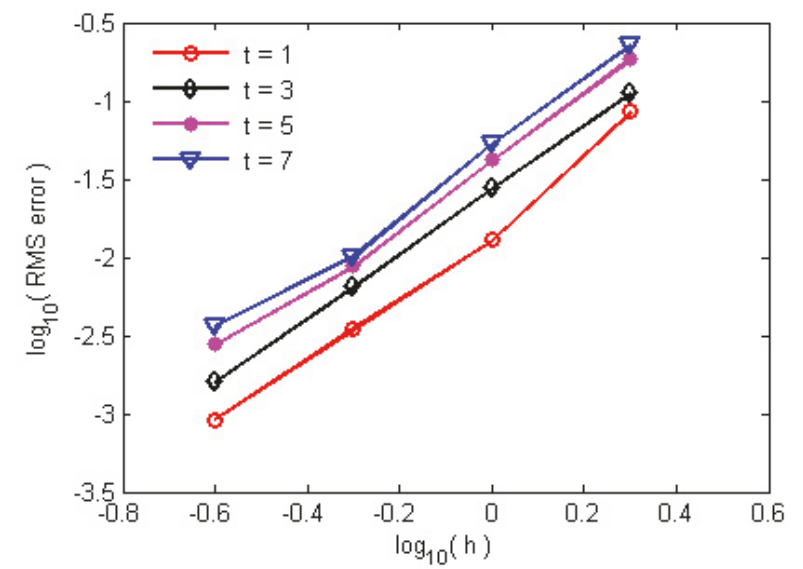

(b)

FIGURE 3: (a) $L_{\infty}$ error and (b) RMS error versus the nodal spacing $h$ for the test problem.

straightness when $t=18$. These graphical results match those obtained by the FEM [7], the FDM [8], the BEM [9, 10], the differential quadrature method (DQM) [11], and the RBF [21].

4.3. Circular Ring Soliton. The circular ring soliton can be simulated by solving the sine-Gordon equation for $\psi\left(x_{1}, x_{2}\right)$ $=1$ and $\beta=0$, with initial conditions [7, 9-11, 21, 23]

$$
\begin{aligned}
& f\left(x_{1}, x_{2}\right)=4 \tan ^{-1} \exp \left[3-\sqrt{x_{1}^{2}+x_{2}^{2}}\right], \\
& g\left(x_{1}, x_{2}\right)=0,
\end{aligned}
$$

$$
x_{1}, x_{2} \in(-7,7)
$$

and boundary condition

$$
\frac{\partial u(\mathbf{x}, t)}{\partial \mathbf{n}}=0, \quad \mathbf{x} \in \Gamma, t \geq 0
$$

Figure 5 depicts the results of this problem for $\tau=0.01$ and $h=0.5$ at times $t=0,5.6,8.4,11.2$ and 12.6. In this figure, both the contours and surface plots of the ring soliton are given in terms of $\sin (u / 2)$. It can be observed from this figure that the ring soliton shrinks at the initial stage $(t=0)$. Then, as $t$ goes on, oscillations and radiations start to form and continue to form up to $t=8.4$. And an expanding phase is observed at $t=5.6$ and after it. The expansion continues until $t=11.2$, where the ring soliton is nearly formed again. When $t=12.6$, the ring soliton appears to be again in its shrinking phase. These graphical results match those obtained by the FEM [7], the BEM [9, 10], the DQM [11], the RBF [21], and the mesh-free reproducing kernel particle Ritz method [23].

4.4. Collision of Two Circular Ring Solitons. The collision of two circular ring solitons can be simulated by solving 

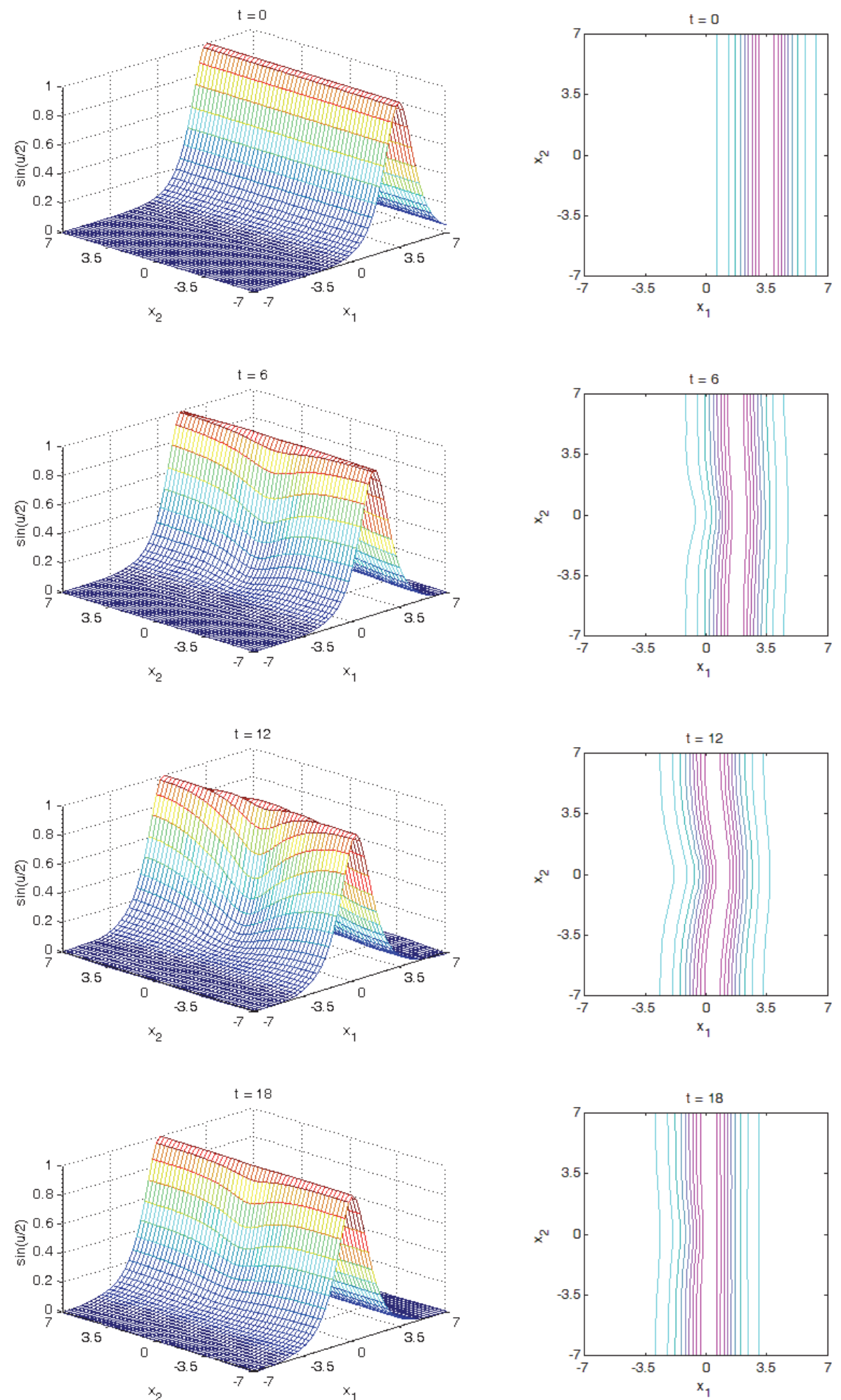

FIGURE 4: Initial condition and numerical solutions at $t=6,12$, and 18 for the line soliton in an inhomogeneous medium: surface plots and contours of $\sin (u / 2)$. 

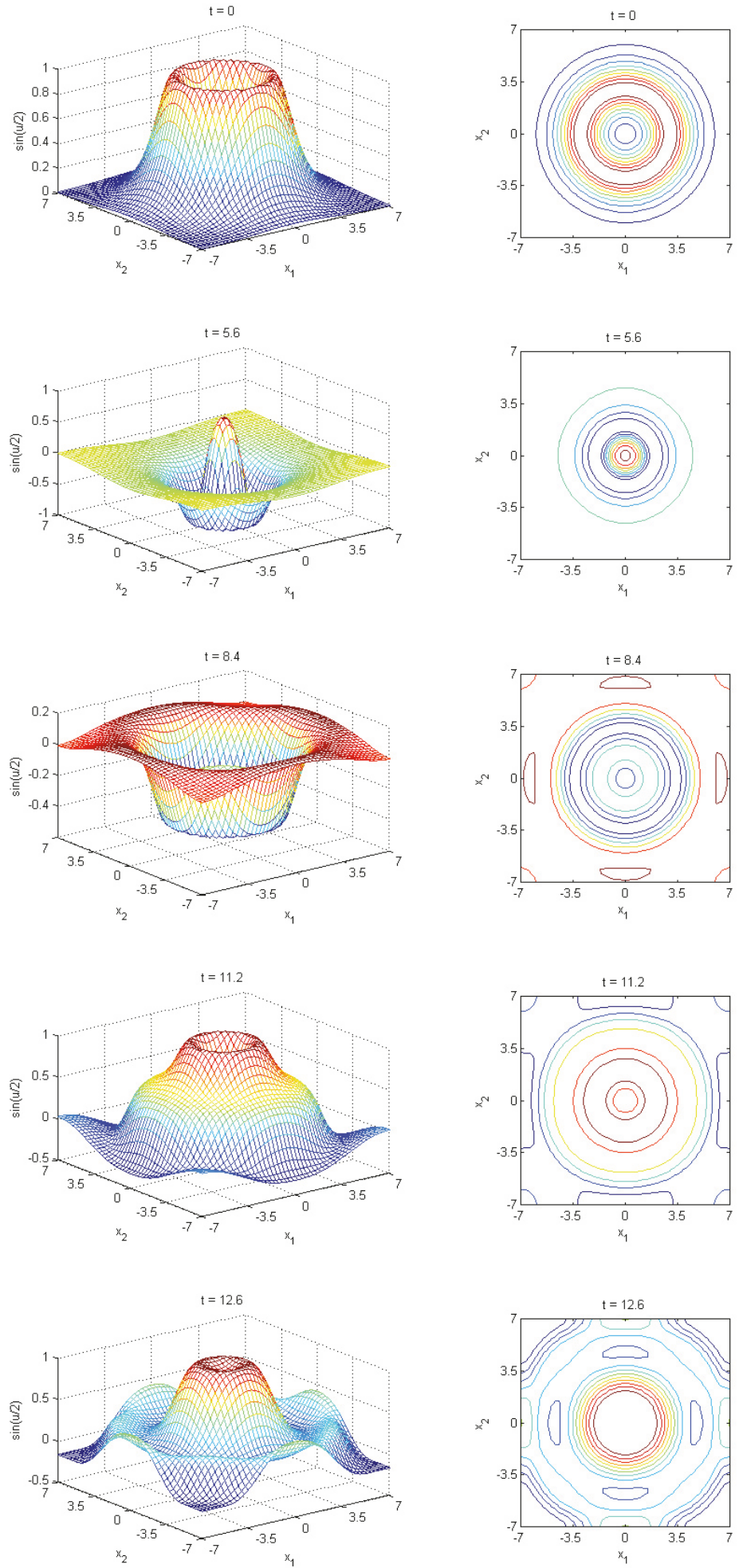

FIGURE 5: Initial condition and numerical solutions at $t=5.6,8.4,11.2$, and 12.6 for the circular ring soliton: surface plots (left panel) and contours (right panel) of $\sin (u / 2)$. 


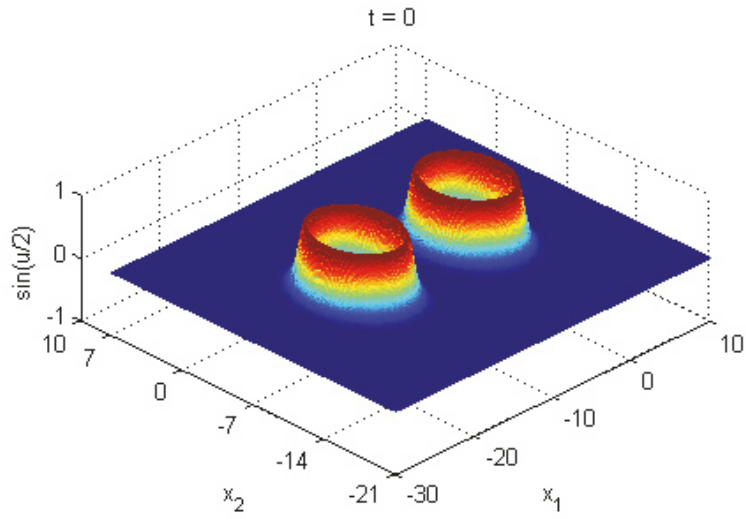

(a)

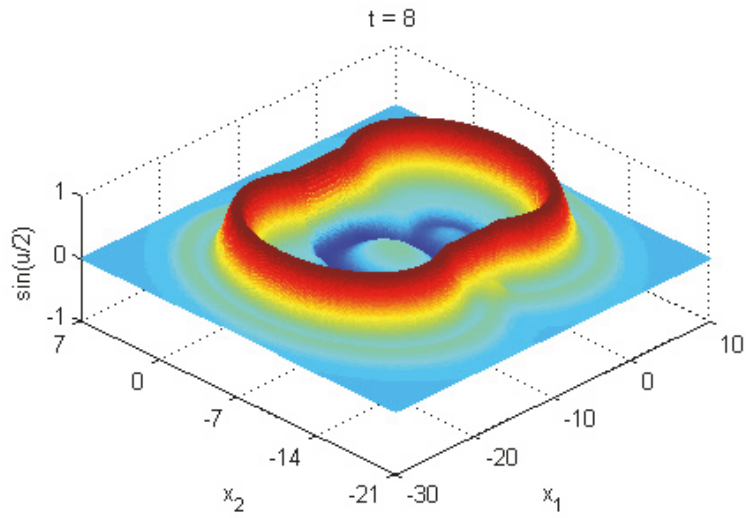

(c)

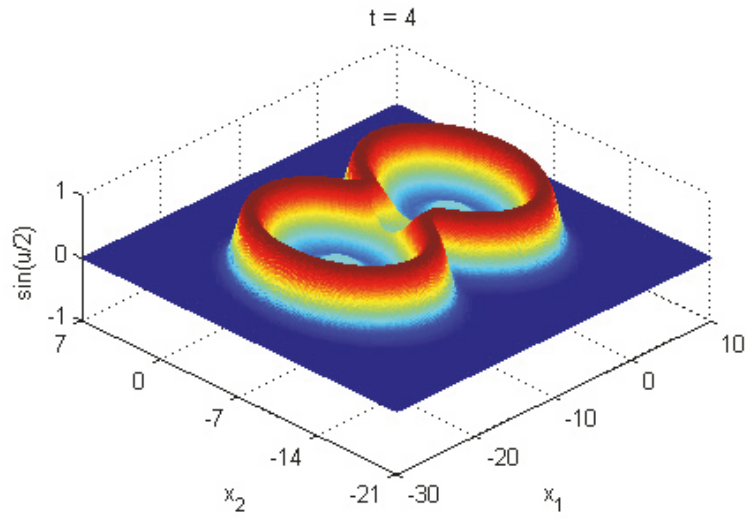

(b)

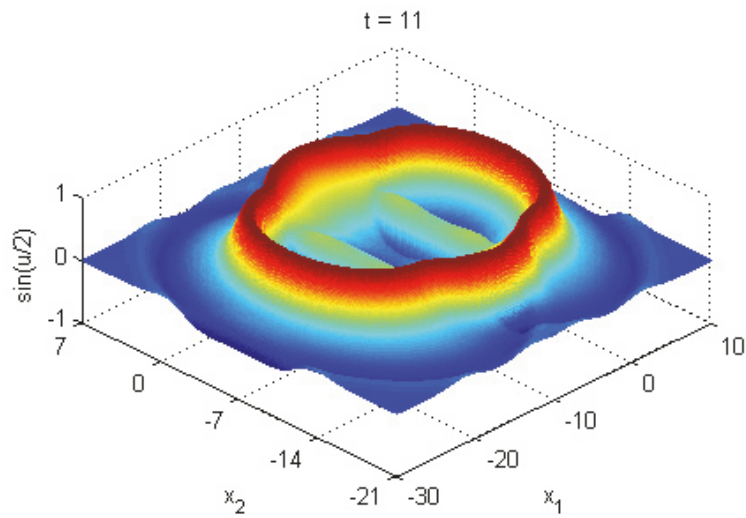

(d)

Figure 6: Results of (a) initial condition $(t=0)$ and numerical solutions at (b) $t=4,(\mathrm{c}) t=8$, and (d) $t=11$ for the collision of two circular ring solitons when $\beta=0$.

the sine-Gordon equation for $\psi\left(x_{1}, x_{2}\right)=1$ with initial conditions $[6,7,10,21]$

$$
\begin{aligned}
& f\left(x_{1}, x_{2}\right) \\
& =4 \tan ^{-1} \exp \left[\frac{\left(4-\sqrt{\left(x_{1}+3\right)^{2}+\left(x_{2}+3\right)^{2}}\right)}{0.436}\right], \\
& g\left(x_{1}, x_{2}\right) \\
& \quad \mathbf{x} \in \Omega \\
& =4.13 \operatorname{sech}\left[\frac{\left(4-\sqrt{\left(x_{1}+3\right)^{2}+\left(x_{2}+3\right)^{2}}\right)}{0.436}\right], \\
& \mathbf{x} \in \Omega
\end{aligned}
$$

and boundary condition

$$
\frac{\partial u(\mathbf{x}, t)}{\partial \mathbf{n}}=0, \quad \mathbf{x} \in \Gamma, t \geq 0
$$

As in $[6,7,10,21]$, the solution is computed by the SBM over $-10 \leq x_{1} \leq 10$ and $-7 \leq x_{2} \leq 7$ and then is expanded at the lines $x_{1}=-10$ and $x_{2}=-7$ by symmetry relations.

When the dissipative parameter $\beta=0.05$, Figure 6 depicts the results for $\tau=0.01$ and $h=0.5$ at times $t=0,4$, 8 , and 11 in terms of $\sin (u / 2)$. The temporal behavior of the soliton wave consists of a shrinking and an expanding phase. We can observe from Figure 6 the collision between two ring solitons in which, as a result of the collision, two ring solitons bounding an annular area emerge into a larger oval ring soliton. These graphical results match those obtained by the FEM [7], the FDM [6], the BEM [10], and the RBF [21].

The dissipative term $\beta$ is expected to slow down the two initial ring solitons to emerge into a larger oval ring soliton. In order to show this, Figure 7 gives the solution at $t=8$ for $\beta=0.05,0.35,1$ and 5. From Figures 6 and 7, we can observe that the ring soliton changes slowly as $\beta$ increases. Additionally, it can be found that with $\beta=0.35$ the solitons at time $t=8$ are close to those given at $t=4$ for $\beta=0$.

\section{Conclusions}

In this paper, a meshless method based on the meshless singular boundary method has been developed to obtain 


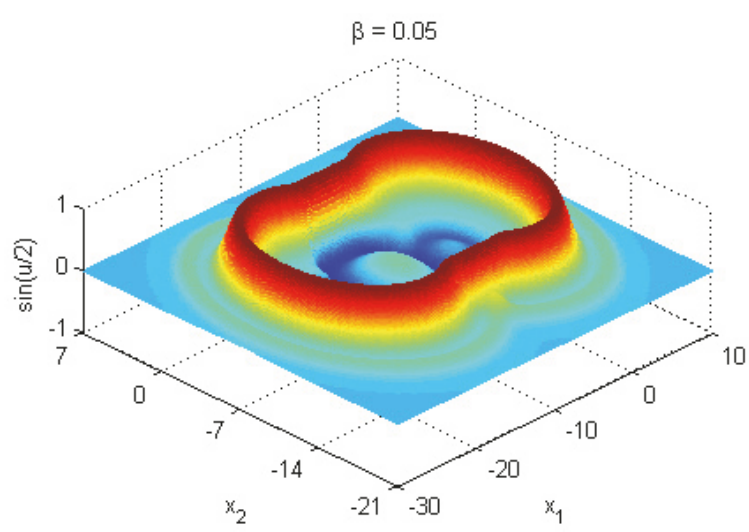

(a)

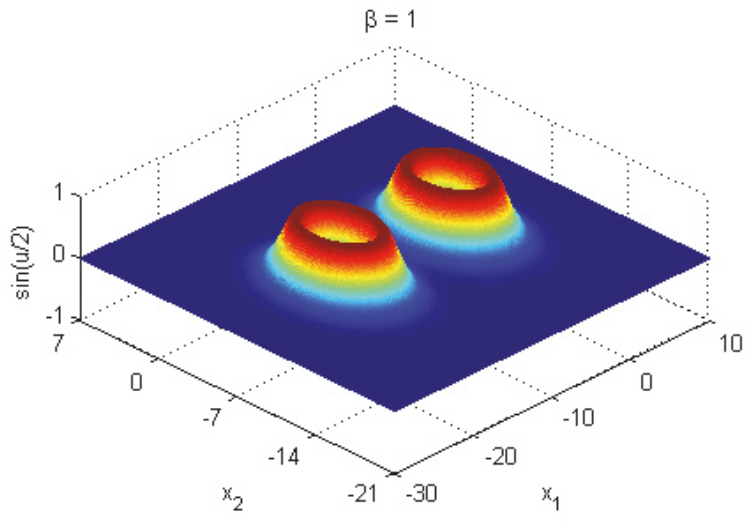

(c)

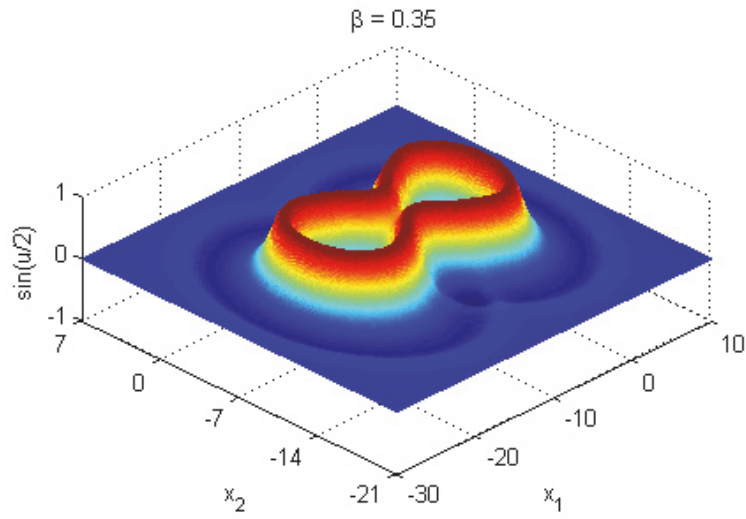

(b)

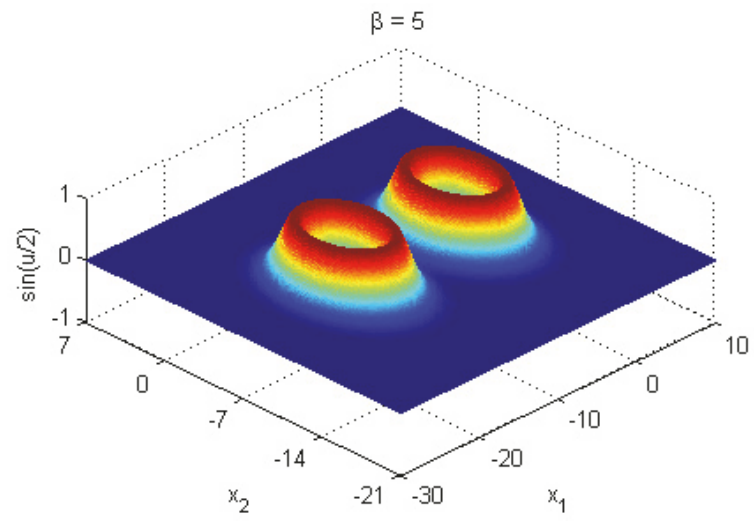

(d)

Figure 7: Numerical solutions at $t=8$ for the collision of two circular ring solitons when (a) $\beta=0.05$, (b) $\beta=0.35$, (c) $\beta=1$, and (d) $\beta=5$.

numerical solutions of the time-dependent nonlinear sineGordon equation. In this method, no mesh is required to discretize the problem domain, and the approximate solution is generated entirely based on scattered nodes. Several numerical examples have been studied to demonstrate the accuracy and efficiency of the method. The numerical results are compared with the results obtained by other methods. For the example with known analytical solution, it is found that the current method obtains more accurate numerical results. For all other examples, the current numerical method produces similar accurate results as those given in [6-11, 21, 23]. Therefore, the SBM is well-suited for solving the nonlinear sine-Gordon equation. However, although numerical results verify the accuracy and the convergence of the method, theoretical proof of stability and convergence is not presented and will be an important research topic for future research.

The current method can be extended to solve other nonlinear equations such as the Klein-Gordon equation, the double sine-Gordon and sinh-Gordon equations, and the hyperbolic telegraph equation. Besides, this meshless method with some modifications is extensible to solve problems in mathematical physics and engineering such as nonlinear optics, plasma physics, solid state physics, and relativistic quantum mechanics. Nevertheless, more research work is required.

\section{Data Availability}

The data used to support the findings of this study are included within the article.

\section{Conflicts of Interest}

The author declares that there are no conflicts of interest regarding the publication of this paper.

\section{References}

[1] P. G. Drazin and R. S. Johnson, Solitons: An Introduction, Cambridge University Press, Cambridge, UK, 1989.

[2] W. Greiner, Relativistic Quantum Mechanics-Wave Equations, Springer, Berlin, Germany, 2000.

[3] G. Leibbrandt, "New exact solutions of the classical sineGordon equation in $2+1$ and $3+1$ dimensions," Physical Review Letters, vol. 41, no. 7, pp. 435-438, 1978.

[4] J. Zagrodzinsky, "Particular solutions of the sine-Gordon equation in $2+1$ dimensions," Physics Letters A, vol. 72, no. 4-5, pp. 284-286, 1979.

[5] A.-M. Wazwaz, "Exact solutions for the generalized sineGordon and the generalized sinh-Gordon equations," Chaos, Solitons \& Fractals, vol. 28, no. 1, pp. 127-135, 2006. 
[6] K. Djidjeli, W. G. Price, and E. H. Twizell, "Numerical solutions of a damped sine-Gordon equation in two space variables," Journal of Engineering Mathematics, vol. 29, no. 4, pp. 347-369, 1995.

[7] J. Argyris, M. Haase, and J. C. Heinrich, "Finite element approximation to two-dimensional sine-Gordon solitons," Computer Methods Applied Mechanics and Engineering, vol. 86, no. 1, pp. 1-26, 1991.

[8] A. G. Bratsos, "The solution of the two-dimensional sineGordon equation using the method of lines," Journal of Computational and Applied Mathematics, vol. 206, no. 1, pp. 251-277, 2007.

[9] M. Dehghan and D. Mirzaei, "The dual reciprocity boundary element method (DRBEM) for two-dimensional sine-Gordon equation," Computer Methods Applied Mechanics and Engineering, vol. 197, no. 6-8, pp. 476-486, 2008.

[10] D. Mirzaei and M. Dehghan, "Boundary element solution of the two-dimensional sine-Gordon equation using continuous linear elements," Engineering Analysis with Boundary Elements, vol. 33, no. 1, pp. 12-24, 2009.

[11] R. Jiwari, S. Pandit, and R. C. Mittal, "Numerical simulation of two-dimensional sine-Gordon solitons by differential quadrature method," Computer Physics Communications, vol. 183, no. 3, pp. 600-616, 2012.

[12] S. Pandit, R. Jiwari, K. Bedi, and M. E. Koksal, "Haar wavelets operational matrix based algorithm for computational modelling of hyperbolic type wave equations," Engineering Computations (Swansea, Wales), vol. 34, no. 8, pp. 2793-2814, 2017.

[13] A. S. Alshomrani, S. Pandit, A. K. Alzahrani, M. S. Alghamdi, and R. Jiwari, "A numerical algorithm based on modified cubic trigonometric B-spline functions for computational modelling of hyperbolic-type wave equations," Engineering Computations (Swansea, Wales), vol. 34, no. 4, pp. 1257-1276, 2017.

[14] A. Verma, R. Jiwari, and S. Kumar, "A numerical scheme based on differential quadrature method for numerical simulation of nonlinear Klein-Gordon equation," International Journal of Numerical Methods for Heat \& Fluid Flow, vol. 24, no. 7, pp. 1390-1404, 2014.

[15] R. Jiwari, "Lagrange interpolation and modified cubic B-spline differential quadrature methods for solving hyperbolic partial differential equations with Dirichlet and Neumann boundary conditions," Computer Physics Communications, vol. 193, pp. 5565, 2015.

[16] G. R. Liu, Meshfree Methods: Moving beyond the Finite Element Method, CRC Press, Boca Raton, Fla, USA, 2009.

[17] Y. M. Cheng, Meshless Methods, Science Press, Beijing, China, 2015.

[18] X. Li and S. Li, "On the stability of the moving least squares approximation and the element-free Galerkin method," Computers \& Mathematics with Applications, vol. 72, no. 6, pp. 15151531, 2016.

[19] X. Li and H. Dong, "The element-free Galerkin method for the nonlinear p-Laplacian equation," Computers \& Mathematics with Applications, vol. 75, no. 7, pp. 2549-2560, 2018.

[20] T. Zhang and X. Li, "A generalized element-free Galerkin method for Stokes problem," Computers \& Mathematics with Applications, vol. 75, no. 9, pp. 3127-3138, 2018.

[21] M. Dehghan and A. Shokri, "A numerical method for solution of the two-dimensional sine-Gordon equation using the radial basis functions," Mathematics and Computers in Simulation, vol. 79, no. 3, pp. 700-715, 2008.
[22] M. Dehghan and A. Shokri, "Numerical solution of the nonlinear Klein-Gordon equation using radial basis functions," Journal of Computational and Applied Mathematics, vol. 230, no. 2, pp. 400-410, 2009.

[23] R. J. Cheng and K. M. Liew, "Analyzing two-dimensional sineGordon equation with the mesh-free reproducing kernel particle Ritz method," Computer Methods Applied Mechanics and Engineering, vol. 245/246, pp. 132-143, 2012.

[24] A. Hussain, S. Haq, and M. Uddin, "Numerical solution of Klein-Gordon and sine-Gordon equations by meshless method of lines," Engineering Analysis with Boundary Elements, vol. 37, no. 11, pp. 1351-1366, 2013.

[25] A. Shokri and A. Habibirad, "A moving Kriging-based MLPG method for nonlinear Klein-Gordon equation," Mathematical Methods in the Applied Sciences, vol. 39, no. 18, pp. 5381-5394, 2016.

[26] X. Li, S. Zhang, Y. Wang, and H. Chen, "Analysis and application of the element-free Galerkin method for nonlinear sine-Gordon and generalized sinh-Gordon equations," Computers \& Mathematics with Applications, vol. 71, no. 8, pp. 1655-1678, 2016.

[27] X. Li and S. Li, "Analysis of the complex moving least squares approximation and the associated element-free Galerkin method," Applied Mathematical Modelling: Simulation and Computation for Engineering and Environmental Systems, vol. 47, pp. 45-62, 2017.

[28] X. Li, "Meshless numerical analysis of a class of nonlinear generalized Klein-Gordon equations with a well-posed moving least squares approximation," Applied Mathematical Modelling: Simulation and Computation for Engineering and Environmental Systems, vol. 48, pp. 153-182, 2017.

[29] C. S. Chen, A. Karageorghis, and Y. S. Smyrlis, The Method of Fundamental Solutions - A Meshless Method, Dynamic Publishers, 2008.

[30] W. Chen and F. Z. Wang, "A method of fundamental solutions without fictitious boundary," Engineering Analysis with Boundary Elements, vol. 34, no. 5, pp. 530-532, 2010.

[31] W. Chen, J.-Y. Zhang, and Z.-J. Fu, "Singular boundary method for modified Helmholtz equations," Engineering Analysis with Boundary Elements, vol. 44, pp. 112-119, 2014.

[32] C. Yang and X. Li, "Meshless singular boundary methods for biharmonic problems," Engineering Analysis with Boundary Elements, vol. 56, pp. 39-48, 2015.

[33] J. Lin, C. Zhang, L. L. Sun, and J. Lu, "Simulation of seismic wave scattering by embedded cavities in an elastic half-plane using the novel singular boundary method," Advances in Applied Mathematics and Mechanics, vol. 10, no. 3, pp. 322-342, 2018.

[34] P. A. Ramachandran and K. Balakrishnan, "Radial basis functions as approximate particular solutions: Review of recent progress," Engineering Analysis with Boundary Elements, vol. 24, no. 7-8, pp. 575-582, 2000.

[35] A. H.-D. Cheng, "Particular solutions of Laplacian, Helmholtztype, and polyharmonic operators involving higher order radial basis functions," Engineering Analysis with Boundary Elements, vol. 24, no. 7-8, pp. 531-538, 2000.

[36] M. Tanaka, V. Slaclek, and J. Sladek, "Regularization techniques applied to boundary element methods," Applied Mechanics Reviews, vol. 47, no. 10, pp. 457-499, 1994. 


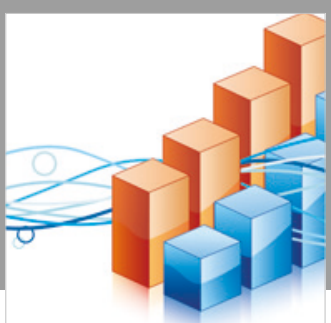

Advances in

Operations Research

\section{-n-m}
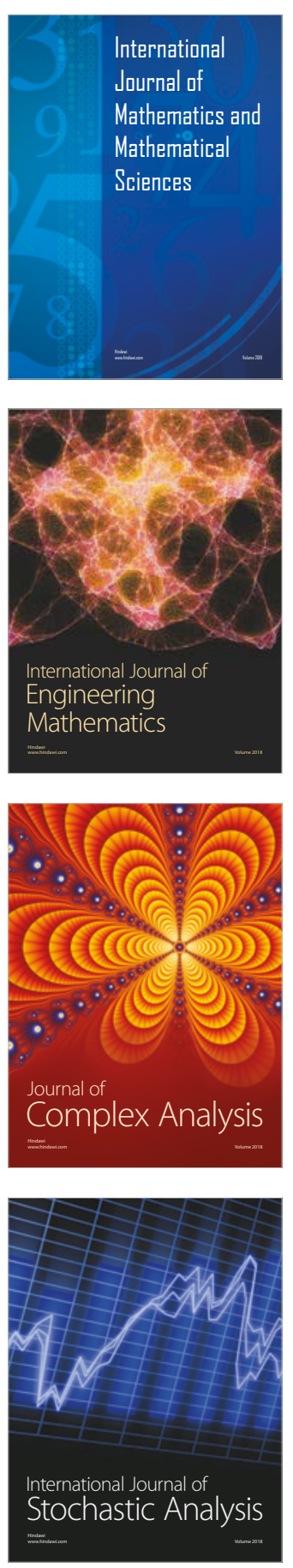
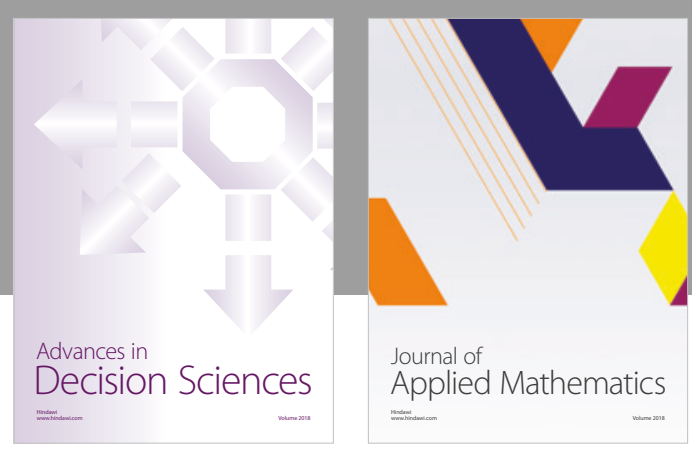

Journal of

Applied Mathematics
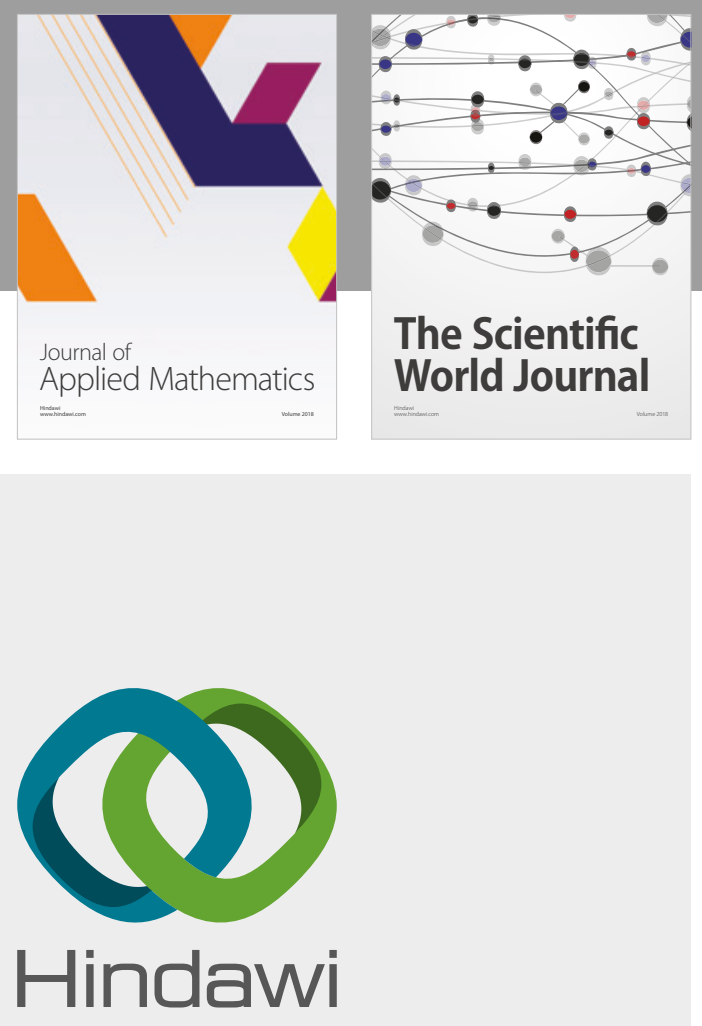

Submit your manuscripts at

www.hindawi.com

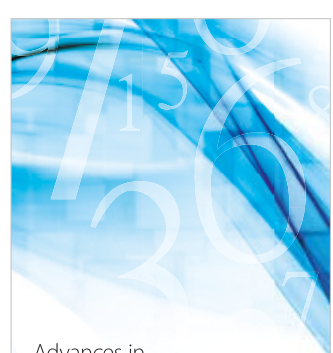

Advances in
Numerical Analysis
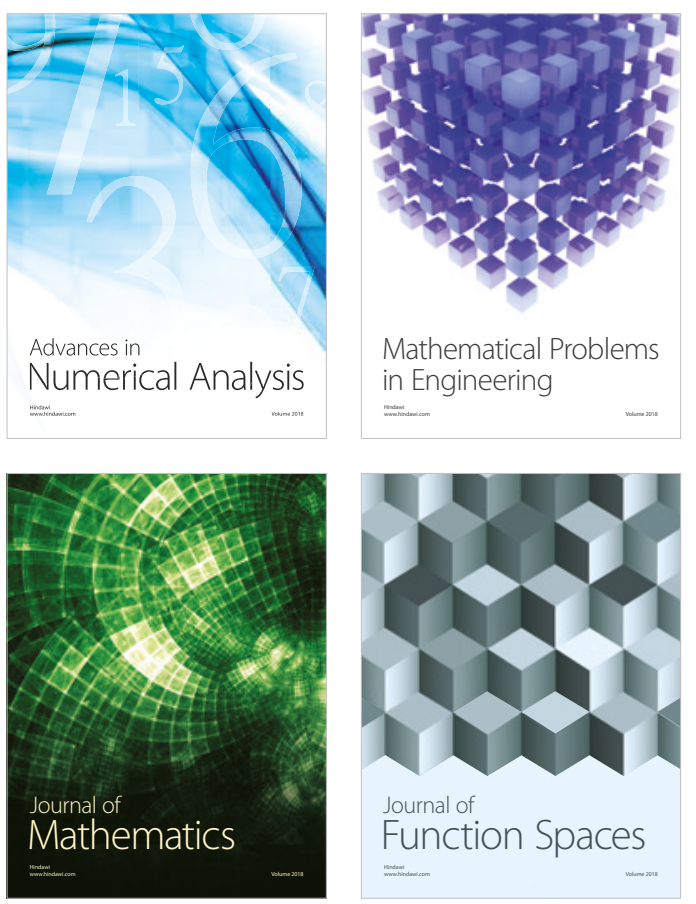

Mathematical Problems in Engineering

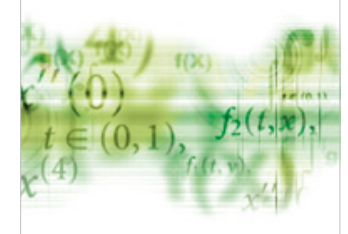

International Journal of

Differential Equations

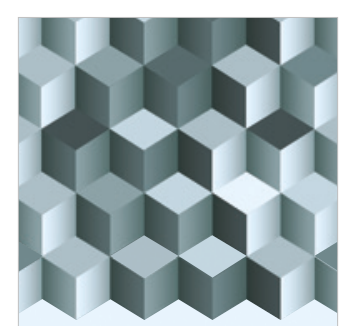

Journal of

Function Spaces

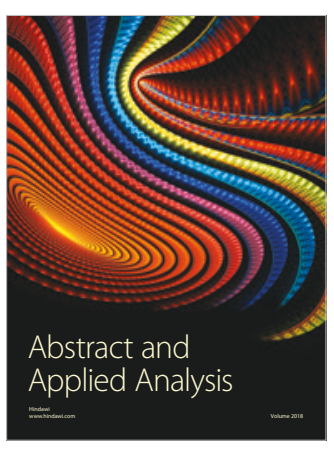

The Scientific

World Journal

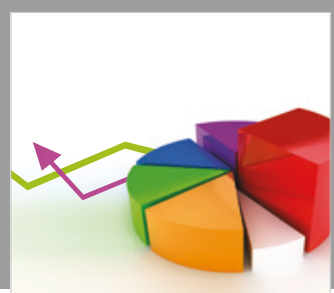

Journal of

Probability and Statistics
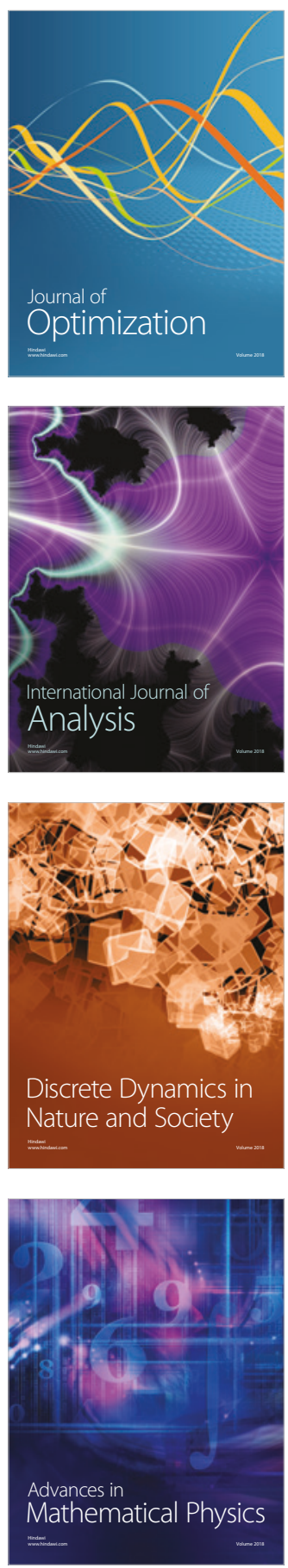